ARTICLE

Received 6 Apr 2011 | Accepted 17 Aug 2011 | Published 13 Sep 2011

DOI: $10.1038 /$ ncomms1484

\title{
A germanate transparent conductive oxide
}

\author{
Hiroshi Mizoguchi', Toshio Kamiya², Satoru Matsuishi² \& Hideo Hosono ${ }^{1,2}$
}

Wide bandgap conductors such as $\ln _{2} \mathrm{O}_{3}$ and $\mathrm{ZnO}$ are used as transparent conducting oxides (TCOs). To date, TCOs are realized using post transition metal cations with largely spread s-orbitals such as $\mathrm{In}^{3+}, \mathrm{Sn}^{4+}, \mathrm{Zn}^{2+}$ and $\mathrm{Cd}^{2+}$. On the other hand, no good electronic conductor has been realized in oxides of $\mathrm{Al}, \mathrm{Si}$ and $\mathrm{Ge}$. Here we report the conversion of an oxide of $\mathrm{Ge}$ into a good electronic conductor by employing the concept of superdegeneracy. We find that cubic $\mathrm{SrGeO}_{3}$, synthesized under high pressure, displays a direct bandgap of $3.5 \mathrm{eV}$, a carrier mobility of $12 \mathrm{~cm}^{2}(\mathrm{Vs})^{-1}$, and conductivities of $3 \mathrm{Scm}^{-1}(\mathrm{DC})$ and $400 \mathrm{Scm}^{-1}$ (optical conductivity). This is the first Ge-based electronic conductive oxide, and expands the family of TCOs from ionic oxides to covalent oxides.

\footnotetext{
${ }^{1}$ Frontier Research Center, Tokyo Institute of Technology, 4259 Nagatsuta, Midori-ku, Yokohama 226-8503, Japan. ${ }^{2}$ Materials and Structures Laboratory, Tokyo Institute of Technology, 4259 Nagatsuta, Midori-ku, Yokohama 226-8503, Japan. Correspondence and requests for materials should be addressed to H.H. (email: hosono@lucid.msl.titech.ac.jp).
} 
T ransparent conducting oxides (TCOs) $)^{1}$, which have a high electrical conductivity and a high optical transparency in visible light, are necessary for a variety of optoelectronic applications. However, high conductivity materials are generally not optically transparent. To date, metal elements such as In, Sn, $\mathrm{Zn}$ and $\mathrm{Cd}$ ('TCO-cations') are essential for TCO formation and form a frontier in the periodic table that separates themselves from the insulating elements such as $\mathrm{Al}, \mathrm{Si}$ and $\mathrm{Ge}$ (the only exception is $12 \mathrm{CaO} \cdot 7 \mathrm{Al}_{2} \mathrm{O}_{3}$ with a special nanocage structure ${ }^{2}$ ). These TCO-cations are post-transition metal cations with electronic configurations of $(n-1) \mathrm{d}^{10} n s^{0}$ ( $n$ is the principal quantum number and should be $\geq 4$ to obtain good electrical conductivities $)^{3-5}$. The spherical spread vacant $s$ orbitals of these metal cations form highly dispersed conduction bands (CBs) with small effective electron masses, and consequently contributes to high electron mobilities. New TCO materials such as $\mathrm{MgIn}_{2} \mathrm{O}_{4}$ have been developed based on this guideline $e^{6-8}$.

However, it is difficult to create a TCO based on the metal cations that are located in the upper right part of the periodic table, because the spatial spread of their vacant $s$ orbitals is small. The small CB dispersion results in a large effective mass and this raises the energy levels of the $\mathrm{CB}$ minimum (CBM), which makes electron doping difficult. In reality, sufficient electronic conduction has yet been obtained for the oxides of metals such as $\mathrm{Al}, \mathrm{Si}$ and $\mathrm{Ge}$.

Herein, we show that cubic- $\mathrm{SrGeO}_{3}$ with the perovskite-type structure(Fig. 1a)isthefirstGe-basedtransparentelectronicconductor.This achievement proposes a new design concept for covalent TCOs, which realizes a deep CBM because of superdegeneracy that appears in high-symmetry crystals of a highly covalent nature.

\section{Results}

Sample structures. Powder samples of undoped and La-doped $\mathrm{SrGeO}_{3}\left(\left(\mathrm{Sr}_{0.95} \mathrm{La}_{0.05}\right) \mathrm{GeO}_{3}\right)$ were synthesized at ambient pressure (AP). These samples were identified as the $\mathrm{AP}-\mathrm{SrGeO}_{3}$ phase by powder X-ray diffraction, as shown in Figure 2a. It has a monoclinic lattice and is composed of an isolated $\left(\mathrm{GeO}_{4}\right)$ ring structure (Fig. 1b). A high-pressure (HP) treatment at $5.5 \mathrm{GPa}$ and $1,100^{\circ} \mathrm{C}$ converted these structures to cubic- $\mathrm{SrGeO}_{3}\left(\mathrm{HP}-\mathrm{SrGeO}_{3}\right)$ with perovskite type ${ }^{9}$ (Fig. 1a), as shown in Figure 2b (a photograph of the material is shown in the inset of Fig. 2e). The observed dense microstructure of the polished surface of the pellet, shown in a scanning electron microscopic (SEM) image in Figure $2 c$ enables us the optical reflectance measurements, as well as carrier transport experiments.

Electrical properties. Figure 2d,e show the temperature dependence of the electrical conductivities and the Seebeck coefficients for the undoped and the La-doped $\mathrm{HP}-\mathrm{SrGeO}_{3}$ samples. The electrical conductivities are nearly temperature independent. Also, the Seebeck coefficients are negative for both the samples, which indicates that these were degenerate $n$-type conductors. The DC conductivity, $\sigma_{\mathrm{DC}}$, at $300 \mathrm{~K}$ for the La-doped sample was $3 \mathrm{Scm}^{-1}$, being consistent with the smaller Seebeck coefficients and the smaller temperature dependence than those of the undoped $\mathrm{HP}-\mathrm{SrGeO}_{3}$.

Optical properties and optical conductivity. Figure 3 shows optical reflectance spectra, complex dielectric functions $\left(\varepsilon^{\star}(\omega)=\varepsilon_{1}(\omega)+j \varepsilon_{2}(\omega)\right.$, where $j$ denotes the imaginary unit), and absorption spectra of the Ge oxides discussed in this paper. From the absorbance spectra shown in the inset of Figure 3a, it is evident that $\mathrm{HP}-\mathrm{GeO}_{2}$ and $\mathrm{HP}-\mathrm{SrGeO}$ have smaller bandgaps than the AP phases. The sharp increase in reflectance at $<0.5 \mathrm{eV}$ can be reproduced using the Drude model with a plasma frequency of $1.0 \mathrm{eV}$ and a momentum relaxation time of $2.0 \mathrm{fs}$, which substantiates that $\mathrm{HP}-\mathrm{SrGeO}_{3}$ has high-density free electrons in the $\mathrm{CB}$. The optical conductivity $\left(\sigma_{\text {opt }}(\omega) \equiv \omega \varepsilon_{2}(\omega)\right)$ at the DC limit, $\sigma_{\text {opt }}(\omega=0)$, of $410 \mathrm{Scm}^{-1}$ was obtained from this fitting. The carrier density $\left(N_{\mathrm{opt}}\right)$ and mobility $\left(\mu_{\mathrm{opt}}\right)$ can be determined from these results by assuming a reasonable electron effective mass, $\mathrm{m}_{\mathrm{e}}{ }^{*}$. In many TCOs, the effective electron masses range between $0.25-0.35 \mathrm{~m}_{e}\left(\mathrm{~m}_{e}\right.$ denotes the rest mass of the electron $)^{10}$. Assuming $\mathrm{m}_{\mathrm{e}}{ }^{*}=0.3 \mathrm{~m}_{\mathrm{e}}$, we obtained
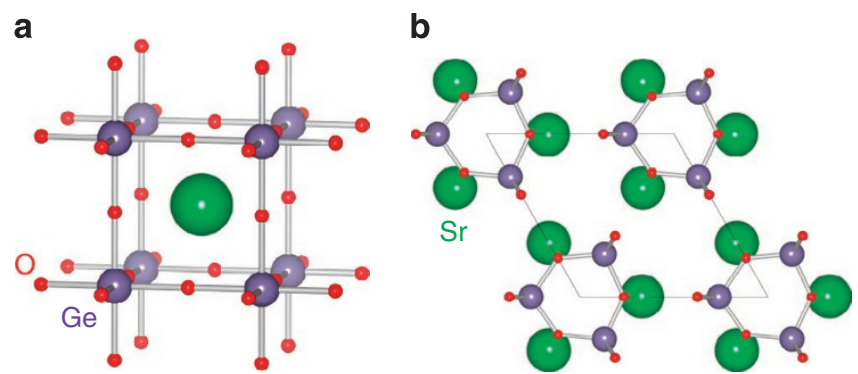

C

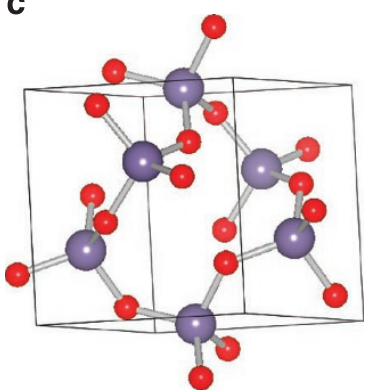

d

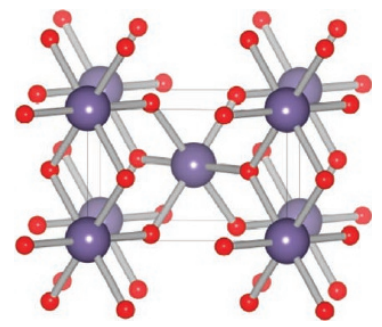

Figure 1 | Crystal structures of Ge oxides. (a) $\mathrm{HP}-\mathrm{SrGeO}_{3}$ with cubic perovskite type. (b) $\mathrm{AP}-\mathrm{SrGeO}_{3}$ composed of isolated rings ${ }^{23,24}$.

(c) AP- $-\mathrm{GeO}_{2}$ has a complex $\alpha$-quartz type structure ${ }^{25}$. (d) $\mathrm{HP}-\mathrm{GeO}_{2}$ takes rutile-type composed of $\mathrm{GeO}_{6}$ octahedra ${ }^{26}$.
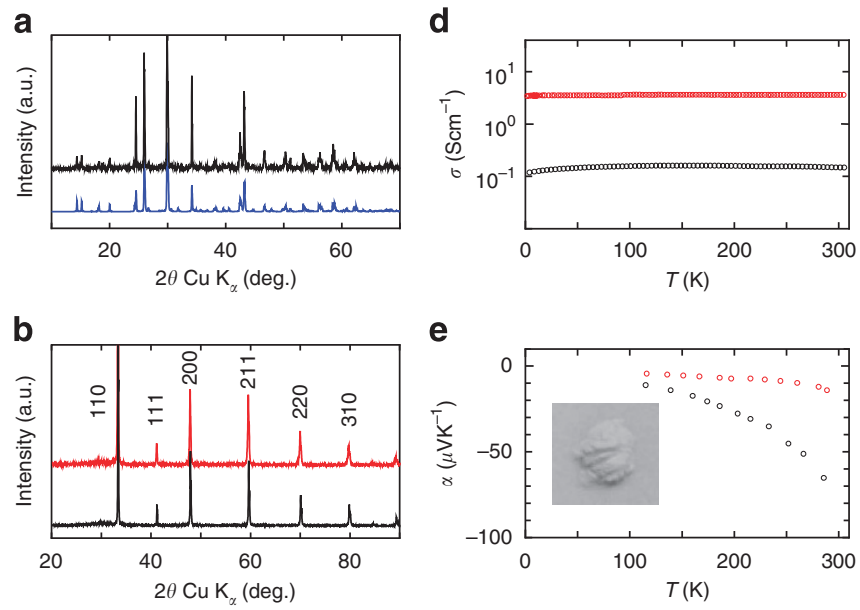

C

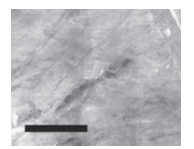

Figure 2 | Powder X-ray diffraction (XRD) patterns and electrical properties. (a) Measured powder XRD pattern of the $\mathrm{SrGeO}_{3}$ sample synthesized at AP (black symbols) along with its simulation pattern (blue symbols). (b) Measured powder XRD patterns of the undoped (black symbols) and La-doped $\mathrm{SrGeO}_{3}$ samples (red symbols) after the HP treatment. (c) SEM image of polished surface of $\mathrm{HP}-\mathrm{SrGeO}_{3}$ pellet. Scale bar shows $3 \mu \mathrm{m}$. (d) Temperature dependence of the electrical conductivities and (e) Seebeck coefficients for the undoped (black symbols) and the La-doped $\mathrm{HP}^{-\mathrm{SrGeO}_{3}}$ (red symbols). Inset to e shows a photograph of the undoped $\mathrm{HP}-\mathrm{SrGeO}_{3}$ powder. 
a

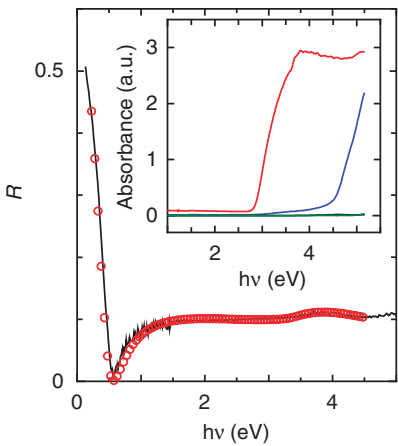

b
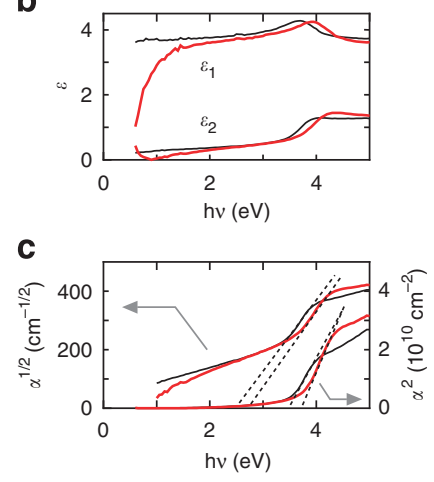

Figure 3 | Bandgaps of $\mathrm{HP}-\mathrm{SrGeO}_{3}$ and related germanium oxides.

(a) Reflectance spectra of La-doped $\mathrm{HP}-\mathrm{SrGeO}_{3}$. The solid line shows the measured data and the red circles show the simulated result using the Drude-Tauc-Lorentz combined model. The inset shows the diffuse reflectance spectra of the powder samples of undoped $\mathrm{AP}-\mathrm{GeO}_{2}$ (the green line), $\mathrm{HP}-\mathrm{GeO}_{2}$ (blue), $\mathrm{AP}-\mathrm{SrGeO}_{3}$ (completely overlapped with the data of $\mathrm{AP}-\mathrm{GeO}_{2}$, the green line) and $\mathrm{HP}-\mathrm{SrGeO}_{3}$ (red). (b) Dielectric functions and (c) absorption spectra for undoped (the black lines) and La-doped $\mathrm{HP}-\mathrm{SrGeO}_{3}$ (the red lines) obtained by spectroscopic ellipsometry. The absorption spectra are plotted for the direct and indirect transition-type semiconductors.

$N_{\text {opt }}=2.2 \times 10^{20} \mathrm{~cm}^{-3}$ and $\mu_{\text {opt }}=11.6 \mathrm{~cm}^{2}(\mathrm{Vs})^{-1}$. We needed to incorporate a $75 \mathrm{~nm}$ thick surface insulating layer to reproduce the dip structure that occurs at $0.55 \mathrm{eV}$, which suggests that the mobile electrons are annihilated in the thin surface region.

Electronic structure. The bandgap values were obtained from an analysis of the spectroscopic ellipsometry data shown in Figure 3b,c. The $\varepsilon_{2}(\omega)$ values show clear absorption structures at $>3 \mathrm{eV}$ for the undoped and the La-doped $\mathrm{SrGeO}_{3}$. For undoped and La-doped $\mathrm{SrGeO}_{3}$, the indirect bandgaps $\left(E_{\text {g,indir }}\right)$, which were estimated from the $\alpha^{0.5}-\mathrm{hv}$ plots, were 2.70 and $2.80 \mathrm{eV}$, respectively. The direct bandgaps $\left(E_{\mathrm{g}, \mathrm{dir}}\right)$ estimated from the $\alpha^{2}-\mathrm{h} v$ plots were 3.45 and $3.70 \mathrm{eV}$, respectively.

We used density functional theory to explain the high electrical conductivity of $\mathrm{HP}-\mathrm{SrGeO}_{3}$. Figure $4 \mathrm{a}$ shows the band structure of $\mathrm{HP}-\mathrm{SrGeO}_{3}$ that indicates that $\mathrm{HP}-\mathrm{SrGeO}_{3}$ is a semiconductor with an indirect bandgap of $2.7 \mathrm{eV}$. Figure $4 \mathrm{~b}$ shows the projected density of states (DOS) for $\mathrm{HP}-\mathrm{SrGeO}_{3}$. Vacant $\mathrm{Sr}$ orbitals do not contribute to the bandgap because their contribution appears only at $>8 \mathrm{eV}$, which is much higher than the CBM (located at $+2.7 \mathrm{eV}$ ). It is thus obvious that $\mathrm{HP}-\mathrm{SrGeO}_{3}$ is a Ge-based electronic conductor. The calculated optical absorption spectra and the DOSs of Ge oxides are shown in Figure 4c,d, respectively. The calculated absorption edge energies agree well with the experimental absorbance spectra shown in the inset of Figure 3a. However, a large discrepancy between the calculated absorption edge $(3.3 \mathrm{eV})$ and bandgap $(2.7 \mathrm{eV}$, extracted from the band structure in Fig. 4a) is found for $\mathrm{HP}-\mathrm{SrGeO}_{3}$.

\section{Discussion}

$\mathrm{HP}-\mathrm{SrGeO}_{3}$ is the first Ge-based transparent electronic conductor with a bandgap of $3.5 \mathrm{eV}$, although $\mathrm{Ge}$ oxides are typical insulators with wide bandgaps $(>5 \mathrm{eV})$. The $\mathrm{HP}-\mathrm{SrGeO}_{3}$ is a well-known phase for geophysicists and has been investigated extensively from geophysical interests ${ }^{11}$; however, no cultivation of electronic functions has, to our knowledge, been examined so far.

To understand the peculiar properties of $\mathrm{HP}-\mathrm{SrGeO}_{3}$, we, first, survey the characteristic features of the electronic structures of the Ge oxides. Ge ions in most of the Ge oxides synthesized at AP take tetrahedral $\mathrm{GeO}_{4}$ sites as shown in Figure 1b,c, and have large band-

gaps (the inset to Fig. 3a). The insulating nature of $\mathrm{AP}-\mathrm{GeO}_{2}$ with the $\alpha$-quartz type structure (Fig. 1c) originates from the large bandgap $(5.1 \mathrm{eV})^{12}$ and its structural flexibility. In this structure, two $\left(\mathrm{GeO}_{4}\right)$ tetrahedra are connected through a twofold coordinated $\mathrm{O}$ ion. The tetrahedral $\mathrm{GeO}_{4}$ is composed of highly covalent $\mathrm{Ge}-\mathrm{O}$ bonds, and this twofold oxygen bridging structure, $\left(\mathrm{GeO}_{4}\right)-\left(\mathrm{GeO}_{4}\right)$, is flexible, which leads to the large bandgap and difficulty in carrier doping because of the ease of structural relaxation on impurity doping. The coordination number of $\mathrm{Ge}^{4+}$ ion increases to six in the HP phases (Fig. 1a,d), and the decrease of the bandgap is clearly observed as seen in the inset of Figure $3 a$.

The right-hand panel of Figure $4 \mathrm{~d}$ shows the band alignment of these materials, which was compiled from the calculated DOSs in the left figure of Figure $4 \mathrm{~d}$. The CBMs of these oxides are composed of $\mathrm{Ge} 4 \mathrm{~s}$ and $\mathrm{O} 2 \mathrm{p}$ orbitals, and the valence band maximums (VBMs) are usually composed of $\mathrm{O} 2 \mathrm{p}$ orbitals. The differences in the VBM energies are as small as $1 \mathrm{eV}$, and therefore, the bandgap differences among these Ge oxides are caused primarily by the difference in the CBM energies.

Next, we describe the origin of the small bandgap of $\mathrm{HP}-\mathrm{SrGeO}_{3}$ based on the concept of superdegeneracy that prohibits hybridization of relevant orbitals at the $\Gamma$ point due to the symmetry and the periodicity in a crystal; that is, the transfer and overlap integrals between an $s$ orbital and two sandwiching $p_{\mathrm{x}}$ orbitals are exactly zero, which leaves the $s-p_{\mathrm{x}}$ bonds nonbonding states at the $\Gamma$ point ${ }^{13-15}$. The CBMs of conventional Ge-based oxides are high, because the Ge $4 \mathrm{~s}$ and $\mathrm{O} 2 \mathrm{p}$ orbitals form covalent chemical bonds and the CBM levels are raised by their resultant anti-bonding nature. However, in $\mathrm{HP}-\mathrm{SrGeO}_{3}$ with cubic perovskite type, the CBMs belong to superdegenerate systems ${ }^{14,15}$ where the translational symmetry strictly prohibits hybridization between the Ge $4 s$ and $\mathrm{O} 2 p$ orbitals at the $\Gamma$ point. This is illustrated in the right-bottom figure of Figure $4 \mathrm{a}$. Consequently, the CBM corresponds to a nonbonding state that is exclusively composed of $\mathrm{Ge} 4 s$ orbitals and its energy level remains unchanged. Regarding the CBM dispersion, the energy level at the $\mathrm{X}$ point (the right-middle figure) is significantly raised, because a $\mathrm{Ge}$ $4 s$ orbital hybridizes with a lobe of an aligned $\mathrm{O} 2 p$ orbital forming an anti-bonding orbital. Therefore, $\mathrm{HP}-\mathrm{SrGeO}_{3}$ has a dispersed $\mathrm{CB}$ with a width of $7 \mathrm{eV}$, which results in a deep CBM energy and the small bandgap of $2.7 \mathrm{eV}$, despite having the largest $\mathrm{Ge}-\mathrm{Ge}$ interionic distance $(0.380 \mathrm{~nm})$ among the four Ge-oxide crystals. The low energy and large dispersion of the CBM in the $\mathrm{HP}-\mathrm{SrGeO}_{3}$ phase makes it possible to realize high-density electron doping and a small effective electron mass in this material.

Finally, we describe the large discrepancy between the calculated absorption edge $(3.3 \mathrm{eV})$ and bandgap $(2.7 \mathrm{eV})$ for $\mathrm{HP}-\mathrm{SrGeO}_{3}$. This discrepancy originates from the fact that $\mathrm{HP}-\mathrm{SrGeO}_{3}$ is an indirect transition-type semiconductor, which is indicated by the band structure shown in Figure 4a. The results obtained in Figures 2d,e and 3 show that the sample has a high density of doped electrons and a high electrical conductivity in addition to transparency (that is, the white colour of the sample seen in the inset of Fig. 2e). In HP$\mathrm{SrGeO}_{3}$, the high conductivity is caused by the deep CBM, which originates from the small indirect bandgap, and the visible transparency is caused by the large direct bandgap $(>3 \mathrm{eV})$.

In summary, we have found that a Ge oxide, cubic $\mathrm{SrGeO}_{3}$, is converted to a good electronic conductor by keeping visible transparency. A low CBM energy and a large $\mathrm{CB}$ dispersion originating from superdegeneracy in the high symmetry of the cubic perovskite structure make it possible for $\mathrm{SrGeO}_{3}$ to have both the high conductivity and the high transparency. On the basis of the present finding, we propose that wide-gap oxides with high-symmetry crystal structures and high covalency are potential candidates for new TCOs irrespective of the absence of a large overlap between the vacant $s$-orbitals of neighbouring metal cations. We expect this to serve as a new design concept for frontier cultivation of TCOs, and 
a

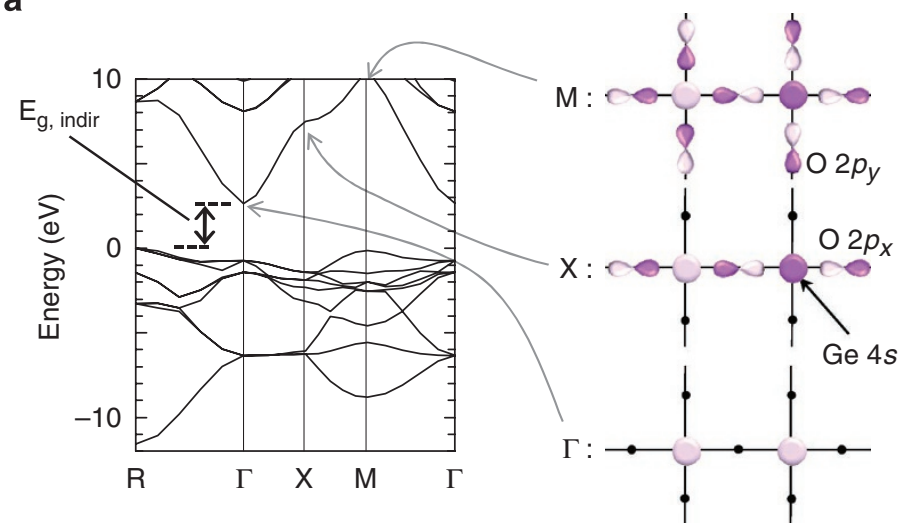

C

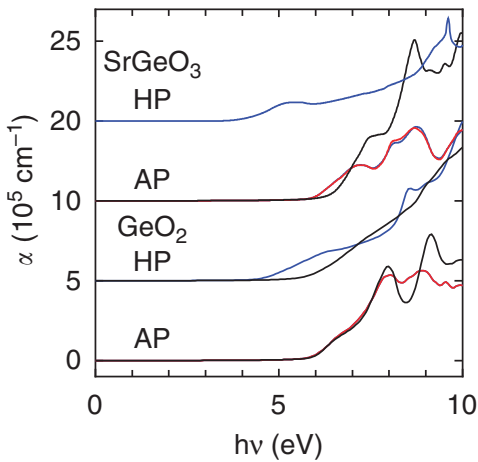

$\mathrm{hv}(\mathrm{eV})$ d

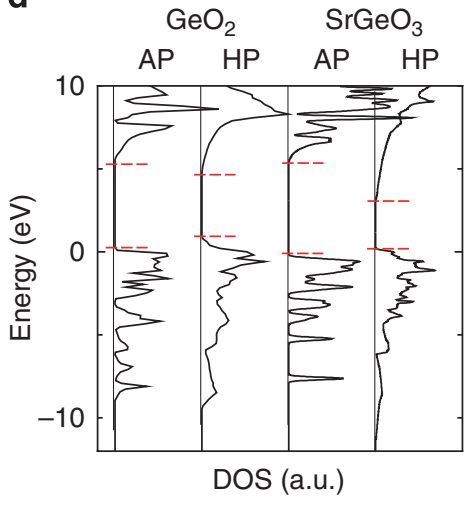

b

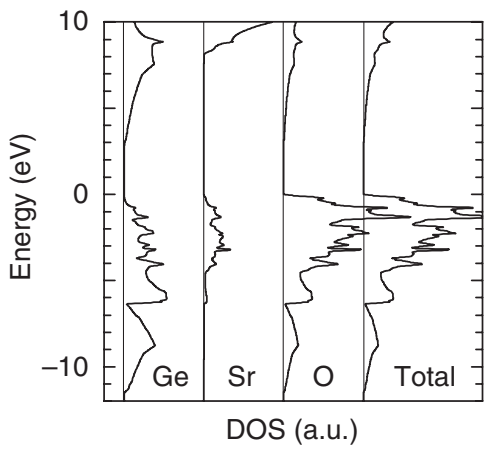

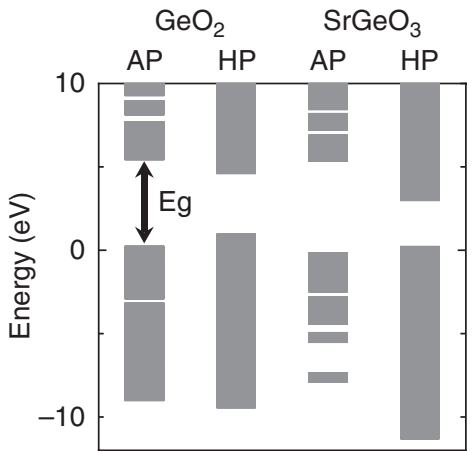

Figure 4 | Calculated electronic structures of germanium oxides. (a) Band structure and illustrations of the corresponding chemical bonds for $\mathrm{HP}-\mathrm{SrGeO}_{3}$. The light and dark purple areas indicate positive and negative phases of the wave functions, respectively. The dots indicate atoms that do not contribute to the chemical bonds. (b) Total and projected DOSs (O, Sr and Ge) for $\mathrm{HP}_{-} \mathrm{SrGeO}$. Those of $\mathrm{Sr}$ and $\mathrm{Ge}$ are expanded by a factor of 5 . (c) Calculated optical absorption spectra for $\mathrm{AP}-\mathrm{GeO}_{2}, \mathrm{HP}-\mathrm{GeO}_{2}, \mathrm{AP}-\mathrm{SrGeO}$ and $\mathrm{HP}-\mathrm{SrGeO}{ }_{3}, \alpha_{x x \prime}, \alpha_{y y}$ and $\alpha_{\mathrm{zz}}$ are represented by the blue, red and black lines, respectively. Each spectrum is shifted by $5 \times 10^{5} \mathrm{~cm}^{-1}$ for clarity. (d) Total DOSs and simplified band alignment for the four Ge-based oxides. The energy is aligned by the $\mathrm{O} 2 \mathrm{~s}$ levels ( -17 to $-21 \mathrm{eV}$ below their respective $\mathrm{E}_{\mathrm{VBM}} \mathrm{s}$ ). The DOSs of the unoccupied states of $\mathrm{HP}^{-\mathrm{SrGeO}} \mathrm{O}_{3}$ are magnified by a factor of 5 . The band edges are shown by the red dashed lines.

it is comparable to a design concept of 'cage conduction band'10,16, which is derived from the discovery of transparent conductive $12 \mathrm{CaO} \cdot 7 \mathrm{Al}_{2} \mathrm{O}_{3}$ (ref. 17).

\section{Methods}

Synthesis. Polycrystalline samples of $\mathrm{SrGeO}_{3}$ were prepared using a solid-state reaction from $\mathrm{SrCO}_{3}, \mathrm{GeO}_{2}$ and $\mathrm{La}_{2} \mathrm{O}_{3}$. Stoichiometric amounts $\left(\mathrm{SrGeO}_{3}\right.$ for undoped, and $\left(\mathrm{Sr}_{0.95} \mathrm{La}_{0.05}\right) \mathrm{GeO}_{3}$ for La-doped $\left.\mathrm{SrGeO}_{3}\right)$ of the reagents were mixed and calcined at $1,200^{\circ} \mathrm{C}$ for $10 \mathrm{~h}$ in air under AP to synthesize the AP-phase $\mathrm{SrGeO}_{3}$. The AP-phase samples were subjected to high-pressure reactions at $5.5 \mathrm{GPa}$ and $1,100^{\circ} \mathrm{C}$ for $1 \mathrm{~h}$, using a belt-type $\mathrm{HP}$ apparatus. AP- and HP-phase $\mathrm{GeO}_{2}\left(\mathrm{AP}-\mathrm{GeO}_{2}\right.$ and $\mathrm{HP}-\mathrm{GeO}_{2}$, respectively) were also synthesized for comparison.

Characterization. Powder X-ray diffraction patterns were obtained using $\mathrm{Cu}$ $\mathrm{K}_{\alpha}$ radiation to determine the crystal structure. The cation stoichiometry was confirmed by X-ray energy dispersive spectroscopy with a Hitachi S-4500 SEM equipped with a Kevex Sigma analyser.

DC electrical conductivity measurements were carried out by the conventional four-probe method. Seebeck coefficient measurements were performed using the static method.

Diffuse reflectance spectra were measured on powder samples over the spectral range of $240-2,600 \mathrm{~nm}$ using $\mathrm{MgO}$ powder as a reference. The data were transformed to absorbance spectra using the Kubelka-Munk relation. Absolute reflectance spectra were measured in the range from $250-2,500 \mathrm{~nm}$ with the spectrophotometer (Hitachi, U-4000) equipped with an absolute specular reflection attachment at an incident angle of $5^{\circ}$ from the normal. IR reflectance spectra were measured using mirror-polished samples and a Fourier transform IR spectrometer (Perkin Elmer, Spectrum One). The incident angle was fixed at $20^{\circ}$ from the normal and an Al mirror was used as a 100\% reflectance standard. Optical dielectric functions were measured by spectroscopic ellipsometry (Jobin-Yvon, UVISEL) at incident/reflection angles of $70^{\circ}$ in the photon energy range $0.6-5.0 \mathrm{eV}$. These data were analysed based on the Drude and Tauc-Lorentz model.

Band structure calculation. The band structures were calculated by hybrid functional level density functional theory using a code VASP 5.2 (ref. 18). We employed PBE0 hybrid functional with a standard mixing parameter of $25 \%$ for the exact-exchange term, because we confirmed that it gave a reasonable bandgap for $\mathrm{ZnO}(3.17 \mathrm{eV}$ for the calculated value and $3.37 \mathrm{eV}$ for the experimental value). Additionally, it gave the closest optical properties to the observed optical properties of the $\mathrm{SrGeO}_{3}$ samples among the different functionals used, that is, PBE96, PBE0 and $\mathrm{HSE}^{19-22}$.

\section{References}

1. Ginley, D. S. \& Bright, C. Transparent conducting oxides. MRS Bull. 25, 15-18 (2000).

2. Kim, S.- W. et al. Metallic state in a lime-alumina compound with nanoporous structure. Nano Lett. 7, 1138-1143 (2007).

3. Robertson, J. Electronic structure of $\mathrm{SnO}_{2}, \mathrm{GeO}_{2}, \mathrm{PbO}_{2}, \mathrm{TeO}_{2}$, and $\mathrm{MgF}_{2}$. J. Phys. C 12, 4767-4776 (1979).

4. Kawazoe, H., Yanagi, H., Ueda, K. \& Hosono, H. Transparent p-type conducting oxides: design and fabrication of $\mathrm{p}-\mathrm{n}$ heterojunctions. MRS Bull. $25,28-36(2000)$.

5. Medvedeva, J. E. Combining optical transparency with electrical Conductivity: challenge and prospects. in Transparent Electronics (eds Facchetti, A. \& Marks, T. J.) (Wiley \& Sons, 2010).

6. Kawazoe, H., Ueda, N., Un'no, H., Omata, T., Hosono, H. \& Tanoue, H. Generation of electron carriers in insulating thin film of $\mathrm{MgIn}_{2} \mathrm{O}_{4}$ spinel by $\mathrm{Li}^{+}$ implantation. J. Appl. Phys. 76, 7935-7941 (1994). 
7. Freeman, A. J., Poeppelmeier, K. R., Mason, T. O., Chan, R. P. H. \& Marks, T. J Chemical and thin film strategies for new transparent conducting oxides. MRS Bull. 25, 45-51 (2000).

8. Cava, R. J. et al. $\mathrm{GaInO}_{3}$ : a new transparent conducting oxide. Appl. Phys. Lett. 64, 2071-2072 (1994).

9. Shimizu, Y., Syono, Y. \& Akimoto, S. High-pressure transformations in $\mathrm{SrGeO}_{3}$, $\mathrm{SrSiO}_{3}, \mathrm{BaGeO}_{3}$, and $\mathrm{BaSiO}_{3}$. High Temp. High Press. 2, 113-120 (1970).

10. Medvedeva, J. E. Averaging of the electron effective mass in multicomponent transparent conducting oxides. Europhys. Lett. 78, 57004-1-6 (2007).

11. Grzechnik, A., McMillan, P. F., Chamberlin, R., Hubert, H. \& Chizmeshya, A. V. G. $\mathrm{SrTiO}_{3}-\mathrm{SrGeO}_{3}$ perovskites obtained at high pressure and high temperature. Eur. J. Solid State Inorg. Chem. 34, 269-281 (1997).

12. Boem, H. F. On the photoconductivity of the vitreous $\mathrm{GeO}_{2}$. J. Non-Cryst. Solids 7, 192-202 (1972)

13. Hughbanks, T. Superdegerate electronic-energy levels in extended structures. J. Am. Chem. Soc. 107, 6851-6859 (1985).

14. Wheeler, R. A., Whangbo, M.- H., Hughbanks, T., Hoffmann, R., Burdett, J. K. \& Albright, T. A. Symmetrical vs asymmetric linear M-X-M linkages in molecules, polymers, and extended networks. J. Am. Chem. Soc. 108, 2222-2236 (1986).

15. Mizoguchi, H., Woodward, P. M., Byeon, S.- H. \& Parise, J. B. Polymorphism in $\mathrm{NaSbO}_{3}$ : structure and bonding in metal oxides. J. Am. Chem. Soc. 126, 3175-3184 (2004)

16. Sushko, P. V., Shluger, A. L., Hayashi, K., Hirano, M. \& Hosono, H. Electron localization and a confined electron gas in nanoporous inorganic electrides. Phys. Rev. Lett. 91, 126401-1-4 (2003).

17. Hayashi, K., Matsuishi, S., Kamiya, T., Hirano, M. \& Hosono, H. Light-induced conversion of an insulating refractory oxide into a persistent electronic conductor. Nature 419, 462-465 (2002).

18. Kresse, G. \& Furthmuller, J. Efficient iterative schemes for ab-initio totalenergy calculations using a plain wave basis set. Phys. Rev. B 54, 11159-11186 (1996).

19. Perdew, J. P., Burke, K. \& Ernzerhof, M. Generalized gradient approximation made simple. Phys. Rev. Lett. 77, 3865-3868 (1996).

20. Perdew, J. P., Ernzerhof, M. \& Burke, K. Rationale for mixing exact exchange with density functional approximations. J. Chem. Phys. 105, 9982-9985 (1996)
21. Heyd, J., Scuseria, G. E. \& Ernzerhof, M. Hybrid functionals based on a screened Coulomb potential. J. Chem. Phys. 118, 8207-8215 (2003).

22. Krukau, A. V., Vydrov, O. A., Izmaylov, A. F. \& Scuseria, G. E. Influence of the exchange screening parameter on the performance of screened hybrid functionals. J. Chem. Phys. 125, 224106-1-5 (2006).

23. Hilmer, W. An X-ray investigation of the strontium germinate $\mathrm{SrGeO}_{3}$. Sov Phys. Cryst. 7, 573-576 (1963).

24. Nishi, F. Strontium metagermanate, $\mathrm{SrGeO}_{3}$. Acta Cryst. C 53, 399-401 (1997).

25. Jorgensen, J. D. Compression mechanism in $\alpha$-quartz structures- $\mathrm{SiO}_{2}$ and $\mathrm{GeO}_{2}$. J. Appl. Phys. 49, 5473-5478 (1978).

26. Bolzan, A. A., Fong, C., Kennedy, B. J. \& Howard, C. J. Structural studies of rutile-type metal dioxides. Acta Cryst. B 53, 373-380 (1997).

\section{Acknowledgements}

This work was supported by the Funding Program for World-Leading Innovative R\&D on Science and Technology (FIRST), Japan. We thank Drs T. Atou, O. Fukunaga and S. Fujitsu at Tokyo Institute of Technology for their experimental supports.

\section{Author contributions}

H.M. performed the sample fabrication, measurements and fundamental data analysis T.K. performed density-functional calculations and optical analyses. S.M. performed optical measurements. H.H. provided strategy and advice for the material exploration. All the authors contributed to discussion on the results for the manuscript.

\section{Additional information}

Competing financial interests: The authors declare no competing financial interests.

Reprints and permission information is available online at http://npg.nature.com/ reprintsandpermissions/

How to cite this article: Mizoguchi, H. et al. A germanate transparent conductive oxide. Nat. Commun. 2:470 doi: 10.1038/ncomms1484 (2011).

License: This work is licensed under a Creative Commons Attribution-NonCommercialShare Alike 3.0 Unported License. To view a copy of this license, visit http:// creativecommons.org/licenses/by-nc-sa/3.0/ 